\title{
Is smaller still better? Walking on the trail of cancer nanomedicine
}

\author{
Davide Prosperi \& Fabio Corsi
}

Surgery Department, Breast Unit, ICS Maugeri, via Maugeri 4, 27100 Pavia, Italy

Department of Biomedical and Clinical Sciences University of Milan, "Luigi Sacco" Hospital, via G. B. Grassi 74, 20157 Milano, Italy.

Dipartimento di Biotecnologie e Bioscienze, Università di Milano-Bicocca, piazza della Scienza 2, 20126 Milano, Italy $(\ldots \ldots \ldots \ldots \ldots \ldots \ldots \ldots)$

Email addresses: davide.prosperi@ unimib.it; fabio.corsi@unimi.it

Only seven years have passed since the previous Special Issue dedicated to nanomedicine has been published in Pharmacological Research. However, impressive advances that have been reached both in terms of knowledge of the factors concurring to the design and development of nanomaterials suitable for biomedical application and in terms of steps made in cancer research moving toward clinical translation of that knowledge. Obviously, such advancements have been accompanied by a change in mentality affecting the modern approaches to many human diseases, particularly cancer. We can assume that what we call cancer may not be merely referred to a pool of degenerative diseases sharing a few encoded features, but rather harnesses a plethora of molecular, methabolic, physiologic and even psychological aspects that contribute to the insurgence, the development and the fate of the disease. It is now evident that multiple weapons will be required to face this longlasting fight, as different kinds of tumors exist and, within each kind of tumor, diverse molecular pathways of proliferation, metastasis and resistance, and diverse interactions with blood and vasculature are involved. In addition, there is now evidence that the same cancer type could 
have different evolutions and treatments may have very different outcomes from one individual to another, which suggest that the future of cancer management will need newly optimized therapeutic settings leading to a precision medicine.

An increasingly detailed understanding of the various aspects of cancer etiology is leading to a revolution in the traditional concept of cancer management. Indeed, besides the conventional "maximum tolerable" approach, a "minimum effective" treatment is being applying to the cure of cancer. Examples of minimum effective treatments in current clinical settings are the conservative surgical intervention on the axilla including considerations on adequate margins in breast cancer; the mini-invasive laparoscopic techniques for colo-rectal and gastric cancer; and a more focused radiation therapy [1]. There is growing interest from oncological research groups in attempting to translate the same concept also to chemotherapy with the aim to reduce the impact of cytotoxic agents and improve the life quality of the patients along and after treatments. However, in this case, the way looks a bit longer. Currently available chemotherapy is mostly based on the use of drugs discovered a few decades ago, which generally exhibit excellent cytotoxic efficacy but is limited by poor biodistribution due to untargeted delivery [2]. In absence of selective targeting, the rationale of the antitumor efficacy of chemotherapeutics is that these drugs principally exert their impact on highly proliferative tissues. However, their antiproliferative activity occurs irrespectively of the nature of the cells, whether they are malignant or not, in other words without a specific action on cancer. Cancer is a complex disease which exploits several alternative cell pathways or interactions with the extracellular microenvironment, with substantial heterogeneity both among-subjects and within-subject [3]. As a result, current chemotherapeutic treatments suffer from suboptimal efficacy, often require high doses to provide effective and durable outcome, which however is paid by patients with severe systemic toxicities [4]. In addition, oncologists need frequently to face inadequate clinical responses to therapy or the occurrence of chemoresistance and severe side effects caused by these drugs, resulting in compromised life quality of patients with consequent poor compliance and modest therapy adherence. 
Novel advances in drug design and formulation are strongly demanded to allow therapeutic agents to be delivered selectively to cancer cells and released in those intracellular compartments in which the drug should exert its antiproliferative action [5]. A first clinical proof of the higher efficacy of targeted therapies has been offered by the introduction of Trastuzumab for HER2positive breast cancers, with a significant improvement of the overall survival and a real change in the natural history of this tumor, previously associated to poor outcomes [6].

A great spur directed toward a personalized, targeted and minimally-invasive medicine is established, but innovative therapeutic agents as well as appropriately updated diagnostic tools are necessary to walk on this path. Nanomedicine represents a great promise to achieve these ambitious goals, providing new targeted strategies to fight the proliferation and migration of cancer cells allowing researchers to combine the development of novel drug delivery systems with higly sensitive diagnostic probes, leading to so-called "theranostic" approaches. This Special Issue dedicated to the recent advances in cancer nanotechnology aims to offer new insights into the fast developing arena of nano-theranostics. Among the thousands of newly proposed nanodrugs and nanodiagnostics, only very few have real potential to move toward a clinical translation. The main purpose of this effort is to try to reduce the gap from current good "nano ideas" to a potential pharmacological strategy.

Despite over 150 clinical trials are going to assess the use of nanodrugs for cancer, a diffusion of nanomedicines in the marketplace is still pending. Updated perspectives from translational research are urgently needed to face the newly discovered aspects of cancer pathology and heterogeneity [7]. Only basic nanotechnology has been recently introduced in the clinical practice, for example using albumin-bound paclitaxel (Abraxane®) or liposomal doxorubicin (Doxil@, Myocet ${ }^{\circledR}$ and Caelyx $®$ ) with encouraging results. However, compelling benefit in terms of overall survival still needs to be demonstrated [8]. To achieve sufficient accumulation at the tumor, clinically approved nanodrugs mainly exploit the enhanced permeability and retention (EPR) effect, broadly considered the "Golden gate" to drug delivery. EPR is based on leaky tumor vasculature that allows the 
extravasation of the nanoparticles in correspondence of the tumor sites [9]. As well described by Arranja et al. in their review article "Tumor-targeted nanomedicines for cancer theranostics", EPR effect does not postulate a factual cancer targeting, provided that no molecular recognition is involved in cell interaction and internalization [ref. n.]. In addition, numerous doubts on the actual potential of EPR effect have been raised after moving from experiments with rodents to clinical trials in humans [ref: Danhier F, J Controlled Rel 2016, 244, 108-121]. These restrictions could at least partially explain why clinically used nanoformulations have certainly reduced toxicity, but have failed in improving significantly the anticancer efficacy of treatments or patient survival. To overcome the major limitations of current chemotherapy, an active drugs targeting would be desirable avoiding off-target delivery as far as possible. Therefore it is of paramount importance to find out specific molecular markers for cancer cells, especially in those cases in which a selective target is challenging to find, such as in the case of triple-negative breast carcinoma. This particular subset of breast cancer lacks overexpression of both hormonal receptors and HER2, making it very hard to define a targeted therapy, frequently resulting in poor prognosis [10]. In their article, Bakrania et al. "Novel targets for paclitaxel nanoformulations: hopes and hypes in triple negative breast cancer" have extensively reviewed all the possible targets which could be exploited by a novel class of paclitaxel-loaded nanoparticles for active targeting toward triple-negative breast cancer suggesting new strategies to be explored for the treatment of this aggressive biological portrait of breast tumor [ref. n.].

The above-mentioned heterogeneity makes it difficult to define universal cell surface targets for each cancer subtype. Moreover, the functionalization of nanoparticles with biologic targeting ligands, such as antibodies, is often laborious and complicated by the fact that antibodies are large molecules with a possible impact on biodistribution and immunogenicity of nanoparticles. Galbiati et al. in their article "Blind targeting in action: from phage display to breast cancer cell targeting with peptide-gold nanoconjugates" propose a clever strategy to achieve selective targeting irrespective of specific knowledge of the relevant molecular target in breast cancer cells. In this 
approach, gold nanoparticles were functionalized with tumor homing peptides, which are produced by randomized amino acid sequences available in free libraries, and recognized by cancer cells. In the study, these nanoconjugates efficiently targeted MCF-7 breast cancer cells, demonstrating a high potential of nanotechonology for specific drug delivery even in the case of a missing molecular target [ref. n.].

Gold nanovectors and other synthetic colloidal nanoparticles have been extensively investigated and proved to be excellent drug delivery systems. However, they present several concerns in regard to a possible clinical translation, as their toxic profile in humans has not been fully elucidated and this remains the main reason why they have not been approved in the clinical practice yet. Therefore, alternative biomimetic nanoparticles derived from "natural" sources are being investigated. Endogenous nanoparticles are composed by physiologically present macromolecules which can form nanostructures with a high potential in terms of drug loading and prolonged activity in biological systems. In their comprehensive review article "Comparison of two endogenous delivery agents in cancer therapy: exosomes and ferritin", Li et al. explore two of the most promising endogenous nanoparticles, namely cellular exosomes and ferritin nanocages, describing their potential and disadvantages [ref. n.]. Exosomes have great drug loading capability, but their separation and differentiation from other natural vesiscles is often difficult. In addition, their isolation is frequently associated to hyperactivation of pro-oncogenic pathways with subsequent concerns about their safety. In contrast, ferritin nanocages are rapidly gaining popularity in drug delivery, because they can exploit the interaction with the transferrin receptor 1, which is overexpressed in several cancer cells and on the endothelial layer of the blood-brain barrier, thus providing an innate active targeting capability without the need for further functionalization [11, 12]. Moreover, a strongly enhanced nuclear delivery of ferritin nanoparticles has been demonstrated with tremendous potential for delivery of DNA-damaging agents [13, ref: Zhang L, Li L, Di Penta A, Carmona U, Yang F, Schops R, et al. A natural nuclei targeting and bioactive delivery nanovector. Adv. Healthcare Mater. 4, 1305-1310 (2015)]. On the other hand, large-scale 
production of ferritin nanocages is difficult at present and their drug loading capability is not optimal. Kooijmans et al. offer a more exosome-oriented view in their review article "Modulation of tissue tropism and biological activity of exosomes and other extracellular vesiscles: new nanotools for cancer treatment", in which exosomes are considered promising strategies for nanodrug delivery, due to their different tropism based on their cellular origin [ref. n.]. Likewise, exosomes can easily penetrate across biological barriers and reach cancer reservoirs such as the central nervous system. In accordance with their physiological function, exosomes can load and deliver genic material such as miRNA and other nucleic acids, which are gaining increasing role in silencing cancer pathways and chemoresistance mechanisms. This property is particularly attractive as delivering nucleic acid in living organisms represents a major challenge due to a rapid degradation of genetic material in the extracellular environment caused by nucleases [14]. In their review article "Nanoparticle-mediated delivery of suicide genes in cancer therapy", Vago et al. report on a few recent advances offered by nanomedicine to deliver suicide gene therapy, which consists in genomic sequences that encode for intracellular expression of toxins leading to programmed cell death. Nanotechnology could overcome current problems encountered with nonspecific viral vectors, especially in terms of safety, allowing for targeted delivery of these toxinecoding genes [ref. n.]. Bypassing or crossing biological barriers is another well known concern in gene delivery, strongly limiting the use of viral vectors for therapy. Nanoparticles could contribute in providing new solutions to these limitations, as described by Wang et al. in their review article "Nanoparticles for cancer gene therapy: recent advances, challenges, and strategies" [ref. n.].

Not only does nanodelivery provide effective tumor targeting, but also might promote a proper drug release into the cell or the tumor microenvironment. Various strategies have been described to achieve stimuli-mediated release of drugs from nanoparticles once internalized in cancer cells, including for example $\mathrm{pH}$-dependent variations [15] or proteolytic enzyme enrichment. Mi et al. in their article "Enzyme-responsive multistage vector for drug delivery to tumor tissue" have explored a novel type of smart release of drugs [ref. n.]. The authors conjugated a substrate of the 
metalloproteinase-2 to polymeric nanoparticles associated to silica microparticles. These microparticles promptly released the polymeric nanoparticles into the tumor microenvironment rich of metalloproteinase-2 as a physiological component of the extracellular matrix. This enzymestimulated drug release from nanoparticles smartly replaced the more commonly utilized $\mathrm{pH}$ dependent strategy exploiting a precise interaction with cancer microenvironment. Another brilliant example of tunable drug release from nanoparticles is reported by Centelles et al. in their article "Focused ultrasound induced hyperthermia accelerates and increases the uptake of anti-Her2 antibodies in a xenograft model", in which hyperthermia fostered by focused ultrasounds was exploited to enhance the accumulation of a targeted antibody in cancer cells in a murine model, with retention of the antibody for several days [ref. n.]. Considering that some nanovectors, such as gold nanoparticles, are suitable for hyperthermic treatment and can be easily functionalized toward specific cancer targets, that work opens new perspectives on multimodal strategies for cancer theranostics. The possibility to finely tune cellular internalization and drug release encourages novel therapeutic approaches against cancer, as elegantly demonstrated by Scott et al. in their article "A pyruvate decarboxylase-mediated therapeutic strategy for mimicking yeast metabolism in cancer cells", in which a cationic lipid-silica nanovector was used to deliver the yeast enzyme pyruvate decarboxylase into cancer cells with subsequent inhibition of lactic acid fermentation and increase in toxic acetaldehyde levels [ref. n.].

Another important ambition of nanomedicine is to improve the solubility, half life and bioavailability of conventional chemotherapy. Several potent cytotoxic and antitubulin drugs, including paclitaxel, broadly employed in current first line clinical settings often suffer from low aqueous solubility, thus necessitating the addition of poorly tolerated excipients during the administration. Two different strategies are suggested to overcome these limitations in two different classes of chemotherapeutics. In their article "Enhanced anti-tumor efficacy of paclitaxel with PEGylated lipidic nanocapsules in presence of curcumin and poloxamer: in vitro and in vivo 
studies", Anwar et al. successfully explored the use of PEGylated lipidic nanocapsules to deliver paclitaxel formulated with curcumin to cancer cells, with a significantly enhanced anticancer efficacy in vivo combined with a controlled drug release and improved bioavailability compared to marketed paclitaxel [ref. n.]. Conversely, Valdepérez et al. propose the use of polymeric nanoparticles to enhance the solubility and aqueous stability of metal-based chemotherapy such as carboplatin or oxaliplatin in their article "Polymer-Coated Nanoparticles: Carrier Platforms for Hydrophobic Water- and Air-Sensitive Metallo-Organic Compounds” [ref. n.].

As above mentioned, nanotechnology is readily expected to impact cancer management by overcoming the severe toxicity of drugs by strongly decreasing the chemotherapy delivery in offtarget organs and by circumventing or even preventing chemoresistance [16]. In their article "Reduced in vivo toxicity of doxorubicin by encapsulation in cholesterol-containing self-assembled nanoparticles", Gonzalez-Fajardo et al. demonstrated the efficacy of polynorbornenecholesterol/polyethylene glycol nanoparticles capable of self-assembling in aqueous media to form nanostructures suitable to deliver cytotoxic drugs such as doxorubicin [ref. n.]. Although passive targeting was exploited in that work, the reported nanoformulation effectively delivered anthracyclines into cancer cells, leading to a strong contraction in cardiotoxicity and relevant adverse side effects. Cardiotoxicity reduction was also the focus of the research by Dhamecha et al. in their article "Doxorubicin loaded gold nanoparticles: implication of passive targeting on anticancer efficacy", suggesting that, when properly designed, also EPR-based passive targeting of nanoparticles could contribute to significantly change the toxicity profile of chemotherapy [ref. n.]. Finally, an attractive yet poorly explored view is offered by Mello et al. in their article "Enhancement of chlorpromazine antitumor activity by Pluronics F127/L81 nanostructured system against human multidrug resistant leukemia", in which micellar nanoformulation of chlorpromazine against chronic myeloid leukemia showed a significant anticancer efficacy together with the capability to escape the multidrug resistance mechanisms mediated by P-glycoprotein, which is a key player in chemoresistance of several cancers [ref. n.]. 
In conclusion, new evidence from recent advances in cancer nanomedicine suggests that nowadays several alternative strategies have become available to target both cancer cells and their microenvironment with smart delivery and controlled release of several classes of drugs, including cytotoxic chemotherapeutics but also miRNAs and other gene-interfering molecules. At present, a few hurdles remain that synthetic nanoparticles need to overcome to receive approval for clinical application, essentially associated to toxicity concerns in humans. Nanovectors based on physiologic-derived biomolecules or biomimetic nanostructures, such as exosomes or ferritins, could be the key to overcome some of these safety issues and to accelerate clinical translation of nanotechnology. As a matter of fact, "small" nanodrugs offer great promise for the insidious challenge of cancer management and healthcare. Research in this field must be encouraged as its clinical translation is strongly demanded.

\section{References}

1. Veronesi U, Stafyla V, Luini A, Veronesi P. Breast cancer: from "maximum tolerable" to "minimum effective" treatment. Front Oncol. 2012 Oct 8;2:125.

2. Joo WD, Visintin I, Mor G. Targeted cancer therapy - are the days of systemic chemotherapy numbered? Maturitas. 2013 Dec;76(4):308-14.

3. Burrell RA, McGranahan N, Bartek J, Swanton C. The causes and consequences of genetic heterogeneity in cancer evolution. Nature. 2013 Sep 19;501(7467):338-45.

4. Conway A, McCarthy AL, Lawrence P, Clark RA. The prevention, detection and management of cancer treatment-induced cardiotoxicity: a meta-review. BMC Cancer. 2015 May 7;15:366.

5. Corsi F, Mazzucchelli S. The potential of protein-based nanocages for imaging and drug delivery. Ther Deliv. 2016;7(3):149-51. 
6. Balduzzi S, Mantarro S, Guarneri V, Tagliabue L, Pistotti V, Moja L, D'Amico R. Trastuzumab-containing regimens for metastatic breast cancer. Cochrane Database Syst Rev. 2014 Jun 12;(6):CD006242.

7. Tanaka T, Decuzzi P, Cristofanilli M, Sakamoto JH, Tasciotti E, Robertson FM, Ferrari M. Nanotechnology for breast cancer therapy. Biomed Microdevices. 2009 Feb;11(1):49-63.

8. Xing M, Yan F, Yu S, Shen P. Efficacy and Cardiotoxicity of Liposomal DoxorubicinBased Chemotherapy in Advanced Breast Cancer: A Meta-Analysis of Ten Randomized Controlled Trials. PLoS One. 2015 Jul 23;10(7):e0133569.

9. Wong AD, Ye M, Ulmschneider MB, Searson PC. Quantitative Analysis of the Enhanced Permeation and Retention (EPR) Effect. $\quad$ PLoS One. $2015 \quad$ May 4;10(5):e0123461.

10. Le Du F, Eckhardt BL, Lim B, Litton JK, Moulder S, Meric-Bernstam F, Gonzalez-Angulo AM, Ueno NT. Is the future of personalized therapy in triple-negative breast cancer based on molecular subtype? Oncotarget. 2015 May 30;6(15):12890-908.

11. Truffi M, Fiandra L, Sorrentino L, Monieri M, Corsi F, Mazzucchelli S. Ferritin nanocages: A biological platform for drug delivery, imaging and theranostics in cancer. Pharmacol Res. 2016 May;107:57-65.

12. Fiandra L, Mazzucchelli S, Truffi M, Bellini M, Sorrentino L, Corsi F. In Vitro Permeation of FITC-loaded Ferritins Across a Rat Blood-brain Barrier: a Model to Study the Delivery of Nanoformulated Molecules. J Vis Exp. 2016 Aug 22;(114).

13. Bellini M, Mazzucchelli S, Galbiati E, Sommaruga S, Fiandra L, Truffi M, Rizzuto MA, Colombo M, Tortora P, Corsi F, Prosperi D. Protein nanocages for self-triggered nuclear delivery of DNA-targeted chemotherapeutics in Cancer Cells. J Control Release. 2014 Dec 28;196:184-96.

14. Muthiah M, Park IK, Cho CS. Nanoparticle-mediated delivery of therapeutic genes: focus on miRNA therapeutics. Expert Opin Drug Deliv. 2013 Sep;10(9):1259-73. 
15. Scheeren LE, Nogueira DR, Macedo LB, Vinardell MP, Mitjans M, Infante MR, Rolim CM. PEGylated and poloxamer-modified chitosan nanoparticles incorporating a lysine-based surfactant for $\mathrm{pH}$-triggered doxorubicin release. Colloids Surf B Biointerfaces. 2016 Feb 1;138:117-27.

16. Mazzucchelli S, Bellini M, Fiandra L, Truffi M, Rizzuto MA, Sorrentino L, Longhi E, Nebuloni M, Prosperi D, Corsi F. Nanometronomic treatment of 4T1 breast cancer with nanocaged doxorubicin prevents drug resistance and circumvents cardiotoxicity. Oncotarget. 2016 Dec 25 [Epub ahead of print]. 